\title{
LINEAR TOPOLOGICAL CLASSIFICATIONS OF CERTAIN FUNCTION SPACES
}

\author{
VESKO M. VALOV
}

\begin{abstract}
Some linear classification results for the spaces $C_{P}(X)$ and $C_{P}^{*}(X)$ are proved.
\end{abstract}

\section{INTRODUCTION}

If $X$ is a space then $C_{P}(X)$ denotes the set of all continuous real-valued functions on $X$ with the topology of pointwise convergence. We write $C_{P}^{*}(X)$ for the subspace of $C_{P}(X)$ consisting of all bounded functions. $R$ stands for the usual space of real numbers, $I-$ for the unit segment $[0,1]$ and $Q$ is the Hilbert cube $I^{\omega}$. If $n \geq 1$ then $\mu^{n}$ denotes the $n$-dimensional universal Menger compactum. Let $X$ be a separable metric space. A separable metric space $Y$ is called an $X$-manifold if $Y$ admits an open cover by sets homeomorphic to open subsets of $X$.

Results in [A1, A2 and Ps] show that the linear topological classification of the spaces $C_{P}(X)$ is very complicated. Below the linear topological classification results for the spaces $C_{P}(X)$ which I know are listed:

(1) Let $X$ and $Y$ be non-zero-dimensional compact polyhedra. Then $C_{P}(X)$ $\sim C_{P}(Y)$ if and only if $\operatorname{dim} X=\operatorname{dim} Y$ [Pv]. Here the symbol " " stands for linear homeomorphism.

(2) If $X$ is a locally compact subset of $R^{n}$ such that $\operatorname{cl}(\operatorname{Int}(X)) \cap\left(R^{n}-X\right) \neq \varnothing$ then $C_{P}(X) \sim C_{P}\left(R^{n}\right)$ [Dr1].

(3) If $X$ is a 1-dimensional compact ANR with finite ramification points or a continuum $X$ is a one-to-one continuous image of $[0, \infty)$ then $C_{P}(X) \sim C_{P}(I)$ [KO].

For topological classification results of the spaces $C_{P}(X)$ see [BGM, BGMP, $\mathrm{GH}$ and $\mathrm{M}]$.

The aim of this paper is to prove the following results:

(4) $C_{P}(X) \sim C_{P}(Q)$ if and only if $X$ is a compact metric space containing a copy of $Q$.

(5) Let $X$ be a subset of $R^{n}$. Then $C_{P}(X) \sim C_{P}\left(I^{n}\right)$ iff $X$ is compact and $\operatorname{dim} X=n$.

Received by the editors February 8, 1989.

1980 Mathematics Subject Classification (1985 Revision). Primary 46E10, 54C35.

Key words and phrases. Linear homeomorphism, function space, manifold. 
(6) $C_{P}(X) \sim C_{P}\left(\mu^{n}\right)$ if and only if $X$ is an $n$-dimensional compact metric space containing a copy of $\mu^{n}$.

(7) $C_{P}(X) \sim C_{P}\left(l_{2}\right)$ provided $X$ is an $l_{2}$-manifold (by $l_{2}$ is denoted the separable Hilbert space).

(8) Let $X$ be one of the spaces $Q, I^{n}$ or $\mu^{n}$, and $Y$ be a locally compact subset of an $X$-manifold. Then $C_{P}(Y) \sim C_{P}(X)^{\omega}$ if and only if $Y$ contains a closed copy of the topological sum $\sum X_{i}$ of infinitely many copies of $X$.

Similar results are also proved for the spaces $C_{P}^{*}(X)$.

I am indebted to A. Dranishnikov and the referee for many valuable comments.

\section{Preliminaries}

All spaces under discussion are Tychonoff and all mappings between topological spaces are continuous. By $L_{P}(X)$ is denoted the dual linear space of $C_{P}(X)$ with the weak (i.e. pointwise) topology. It is known that

$$
L_{P}(X)=\left\{\sum_{i=1}^{k} a_{i} \delta_{x_{i}}: a_{i} \in R-(0) \text { and } x_{i} \in X \text { for each } i \leq k\right\} .
$$

Here $\delta_{x}$ is the Dirac measure at the point $x \in X$. We denote

$$
P_{\infty}(X)=\left\{\sum_{i=1}^{k} a_{i} \delta_{x_{i}}: a_{i} \in(0,1) \text { for each } i \text { and } \sum_{i=1}^{k} a_{i}=1\right\}
$$

and $\operatorname{supp}(l)=\left(x_{1}, \ldots, x_{k}\right)$, where $l=\sum_{i=1}^{k} a_{i} \delta_{x_{i}} \in L_{P}(X)$.

Let $A$ be a closed subset of a space $X$. Consider the following conditions:

(i) There is a continuous linear extension operator $u$ : $C_{P}(A) \rightarrow C_{P}(X)$ (recall that $u: C_{P}(A) \rightarrow C_{P}(X)$ is an extension operator if $u(f) \mid A=f$ for every $\left.f \in C_{P}(A)\right)$;

(ii) There is a continuous linear extension operator $u: C_{P}(A) \rightarrow C_{P}(X)$ and a positive constant $c$ such that $\|u(f)\| \leq c$. $\|f\|$ for every $f \in C_{P}^{*}(A)$. Here $\|f\|$ is the supremum norm of $f$;

(iii) There is a regular extension operator $u: C_{P}(A) \rightarrow C_{P}(X)$ i.e. a continuous linear extension operator $u$ with $u\left(1_{A}\right)=1_{X}$ and $u(f) \geq 0$ provided $f \geq 0$.

$A$ is said to be $l$-embedded (resp., $l^{*}$-embedded) in $X$ if the condition (i) (resp., the condition (ii)) holds. If (iii) is satisfied then $A$ is called strongly $l$ embedded in $X$. Dugundji [D] proved that every closed subset of a metric space $X$ is strongly $l$-embedded in $X$ (he did not state this explicitly in this form). It is known (see [AC , Drl]) that $A$ is $l$-embedded (resp., strongly $l$-embedded) in $X$ if and only if there is a mapping $r: X \rightarrow L_{P}(A)$ (resp., $r: X \rightarrow P_{\infty}(A)$ ) such that $r(x)=\delta_{x}$ for every $x \in A$. Such a mapping will be called an $L_{P}$-valued (resp., a $P_{\infty}$-valued) retraction. Every $L_{P}$-valued retraction $r: X \rightarrow L_{P}(A)$ defines a continuous linear extension operator $u_{r}: C_{P}(A) \rightarrow C_{P}(X)$ by setting 
$u_{r}(f)(x)=r(x)(f)$. If the operator $u_{r}$ satisfies the condition (ii), $r$ is said to be a bounded $L_{P}$-valued retraction.

Let $u: C_{P}(A) \rightarrow C_{P}(X)$ be a continuous linear extension operator. Then the mapping $v(f, g)=u(f)+g$ is a linear homeomorphism from $C_{P}(A) \times$ $C_{P}(X ; A)$ onto $C_{P}(X)$, where

$$
C_{P}(X ; A)=\left\{g \in C_{P}(X): g \mid A=0\right\} .
$$

Analogously, if $A$ is $l^{*}$-embedded in $X$ then $C_{P}^{*}(A) \times C_{P}^{*}(X ; A)$ is linearly homeomorphic to $C_{P}^{*}(X)$.

Let $\mathscr{K}$ be a family of bounded subsets of a space $X$ (i.e. $f \mid K$ is bounded for every $K \in \mathscr{K}$ and $\left.f \in C_{P}(X)\right)$ and $E$ be a linear topological subset of $C_{P}(X)$. Then we set

$$
\left(\prod E\right)_{\mathscr{H}}=\left\{\left(f_{1}, \ldots, f_{n}, \ldots\right) \in E^{\omega}: \lim _{n}\left\|f_{n}\right\|_{K}=0 \text { for every } K \in \mathscr{K}\right\}
$$

and

$$
\left(\prod E\right)_{\mathscr{K}}^{*}=\left\{\left(f_{1}, \ldots, f_{n}, \ldots\right) \in\left(\prod E\right)_{\mathscr{K}}: \sup _{n}\left\|f_{n}\right\|<\infty\right\} .
$$

$(\Pi E)_{\mathscr{K}}$ and $(\Pi E)_{\mathscr{H}}^{*}$ are considered as topological linear subspaces of $C_{P}(X)^{\omega}$. We write $\left(\prod E\right)_{b}$ and $\left(\prod E\right)_{b}$ (resp. $\left(\prod E\right)_{c}$ and $\left.\left(\prod E\right)_{c}^{*}\right)$ if $\mathscr{K}$ is the family of all bounded (resp., of all compact) subsets of $X$. In the above notations $\|f\|_{K}$ stands for the set $\sup \{|f(X)|: x \in K\}$. Let us note that if $X$ is pseudocompact and $E$ is a linear subset of $C_{P}(X)$, the space

$$
\left(\prod E\right)_{0}=\left\{\left(f_{1}, \ldots, f_{n}, \ldots\right) \in E^{\omega}: \lim _{n}\left\|f_{n}\right\|=0\right\}
$$

is considered in $[\mathrm{GH}]$.

We need also the following notion: a space $X$ is said to be a $k_{R}$-space [N] if every function $f: X \rightarrow R$ is continuous provided that $f \mid K$ is continuous for each compact subset $K$ of $X$.

\section{LiNEAR tOPOLOGICAL CLASSIFICATIONS OF $C_{P}(X)$}

2.1 Lemma. Let $A$ be a strongly l-embedded (resp., l-embedded or $l^{*}$-embedded) subset of a space $X$. Then $A \times Y$ is strongly l-embedded (resp., l-embedded or $l^{*}$-embedded) in $X \times Y$ for every space $Y$.

Proof. Suppose $A$ is strongly $l$-embedded in $X$. So, there exists a $P_{\infty}$-valued retraction $r_{1}: X \rightarrow P_{\infty}(A)$. Define a mapping $r: X \times Y \rightarrow P_{\infty}(A \times Y)$ by setting

$$
r(x, y)=\sum_{i=1}^{k} a_{i} \delta_{\left(x_{i}, y\right)}, \quad \text { where } r_{1}(x)=\sum_{i=1}^{k} a_{i} \delta_{x_{i}} .
$$

It is easily shown that $r$ is a $P_{\infty}$-valued retraction. Thus, $A \times Y$ is strongly $l$-embedded in $X \times Y$. One can also prove that $r$ is a (bounded) $L_{P}$-valued retraction provided $r_{1}$ is a (bounded) $L_{P}$-valued retraction. Hence, if $A$ is $l$ (resp., $l^{*}$ )-embedded in $X$ then $A \times Y$ is $l$ (resp., $l^{*}$ )-embedded in $X \times Y$. 
2.2 Lemma. Let $A$ be an $l^{*}$-embedded subset of a space $X$. Then $\left(\prod C_{P}(X)\right)_{b}$ is linearly homeomorphic to $\left(\prod C_{P}(A)\right)_{b} \times\left(\prod C_{P}(X ; A)\right)_{b}$.

Proof. Let $u: C_{P}(A) \rightarrow C_{P}(X)$ be a continuous linear extension operator such that $\|u(f)\| \leq c .\|f\|$ for every $f \in C_{P}^{*}(A)$, where $c>0$. Since $\|f\|=\infty$ provided $f \in C_{P}(A)-C_{P}^{*}(A)$, the inequality $\|u(f)\| \leq c .\|f\|$ holds for every $f \in C_{P}(A)$. Then the mapping $r: X \rightarrow L_{P}(A)$, defined by $r(x)(f)=u(f)(x)$, is an $L_{P}$-valued retraction. Consider the linear homeomorphism $v$ from $C_{P}(A) \times$ $C_{P}(X ; A)$ onto $C_{P}(X), v(f, g)=u(f)+g$. Suppose $\left(f_{1}, \ldots, f_{n}, \ldots\right) \in$ $C_{P}(A)^{\omega}$ and $\left(g_{1}, \ldots, g_{n}, \ldots\right) \in C_{P}(X ; A)^{\omega}$. Put

$$
H(K)=\mathrm{cl}_{A}(\bigcup\{\operatorname{supp}(r(x)): x \in K\}),
$$

where $K$ is a subset of $X$. Obviously, $\left\|u\left(f_{n}\right)\right\|_{K} \leq c \cdot\left\|f_{n}\right\|_{H(K)}$ for every $n \in N$. By a result of Arhangel'skii [A2], $H(K)$ is a bounded subset of $A$ provided $K$ is a bounded subset of $X$. Hence, $\left(f_{1}, \ldots, f_{n}, \ldots\right) \in\left(\prod C_{P}(A)\right)_{b}$ if and only if $\left(u\left(f_{1}\right), \ldots, u\left(f_{n}\right), \ldots,\right)$ belongs to $\left(\prod C_{P}(X)\right)_{b}$. Consequently, $\left(v\left(f_{1}, g_{1}\right), \ldots, v\left(f_{n}, g_{n}\right), \ldots\right)$ belongs to $\left(\prod C_{P}(X)\right)_{b}$ if $\left(g_{1}, \ldots, g_{n}, \ldots\right) \in$ $\left.\left(\prod C_{P}(X ; A)\right)\right)_{b}$ and $\left(f_{1}, \ldots, f_{n}, \ldots\right) \in\left(\prod C_{P}(A)\right)_{b}$. Suppose

$$
\left(v\left(f_{1}, g_{1}\right), \ldots, v\left(f_{n}, g_{n}\right), \ldots\right) \in\left(\prod C_{P}(X)\right)_{b} .
$$

Then $\left(f_{1}, \ldots, f_{n}, \ldots\right) \in\left(\prod C_{P}(A)\right)_{b}$ because $v\left(f_{n}, g_{n}\right) \mid A=f_{n}$ for every $n$. Therefore $\left(u\left(f_{1}\right), \ldots, u\left(f_{n}\right), \ldots\right) \in\left(\prod C_{P}(X)\right)_{b}$. So we have $\left(g_{1}, \ldots, g_{n}\right.$, $\ldots) \in\left(\prod C_{P}(X ; A)\right)_{b}$. Thus, $\left(v\left(f_{1}, g_{1}\right), \ldots, v\left(f_{n}, g_{n}\right), \ldots\right)$ belongs to $\left(\Pi C_{P}(X)\right)_{b}$ iff $\left(g_{1}, \ldots, g_{n}, \ldots\right) \in\left(\prod C_{p}(X ; A)\right)_{b}$ and $\left(f_{1}, \ldots, f_{n}, \ldots\right) \in$ $\left(\prod C_{P}(A)\right)_{b}$. Hence, the formula $v_{0}\left(\left(f_{1}, \ldots, f_{n}, \ldots\right),\left(g_{1}, \ldots, g_{n}, \ldots\right)\right)=$ $\left(v\left(f_{1}, g_{1}\right), \ldots, v\left(f_{n}, g_{n}\right) \ldots\right)$ defines a linear mapping from $\left(\prod C_{P}(A)\right)_{b} \times$ $\left(\prod C_{P}(X ; A)\right)_{b}$ onto $\left(\prod C_{P}(X)\right)_{b}$ which is a homeomorphism.

2.3 Lemma. Let $A$ be an $l^{*}$-embedded subset of a space $X$. If every closed and bounded subset of $A$ is compact then $\left(\prod C_{P}(X \times Y)\right)_{c} \sim\left(\Pi C_{P}(A \times Y)\right)_{c} \times$ $\left(\Pi C_{P}(X \times Y ; A \times Y)\right)_{c}$ for any space $Y$.

Proof. Let $u_{1}: C_{P}(A) \rightarrow C_{P}(X)$ be a continuous linear extension operator such that $\left\|u_{1}(f)\right\| \leq c .\|f\|$ for every $f \in C_{P}^{*}(A)$, where $c>0$, and $r_{1}: X \rightarrow L_{P}(A)$ be defined by $r_{1}(x)(f)=u_{1}(f)(x)$. Obviously, $r_{1}$ is an $L_{P}$-valued retraction. For a given space $Y$ the equality $r(x, y)=\sum_{i=1}^{k} a_{i} \delta_{\left(x_{i}, y\right)}$, where $r_{1}(x)=$ $\sum_{i=1}^{k} a_{i} \delta_{x_{i}}$, defines an $L_{P}$-valued retraction from $X \times Y$ into $L_{P}(A \times Y)$. Next, set $u(f)(x, y)=r(x, y)(f)$ for every $(x, y) \in X \times Y$ and $f \in C_{P}(A \times Y)$. It is easily shown that $u: C_{P}(A \times Y) \rightarrow C_{P}(X \times Y)$ is a continuous linear extension operator. 
Claim 1. $\|u(f)\| \leq c .\|f\|$ for every $f \in C_{P}^{*}(A \times Y)$.

Fix a point $(x, y) \in X \times Y$ and an $f \in C_{P}^{*}(A \times Y)$. It follows from the definition of $u$ that

$$
u(f)(x, y)=\sum_{i=1}^{k} a_{i} f\left(x_{i}, y\right), \quad \text { where } r_{1}(x)=\sum_{i=1}^{k} a_{i} \delta_{x_{i}} .
$$

So, $|u(f)(x, y)| \leq \sum_{i=1}^{k}\left|a_{i}\right| \cdot\|f\|$. Take a function $g \in C_{P}^{*}(A)$ with $\|g\|=1$ and $g\left(x_{i}\right)=\operatorname{sgn}\left(a_{i}\right)$ for each $i=1, \ldots, k$. Then $u_{1}(g)(x)=r_{1}(x)(g)=$ $\sum_{i=1}^{k}\left|a_{i}\right|$. Since $\left\|u_{1}(g)\right\| \leq c .\|g\|$, we have $\sum_{i=1}^{k}\left|a_{i}\right| \leq c$. Hence, $|u(f)(x, y)|$ $\leq c .\|f\|$. Claim 1 is proved.

Claim 2. For every compact subset $K$ of $X \times Y$ the set

$$
H(K)=\mathrm{cl}_{A \times Y}(\bigcup\{\operatorname{supp}(r(x, y)):(x, y) \in K\}),
$$

is also compact.

Let $n_{X}: X \times Y \rightarrow X$ and $n_{Y}: X \times Y \rightarrow Y$ be the natural projections. Then $n_{X}(K)$ and $n_{Y}(K)$ are compact subsets of $X$ and $Y$ respectively. By a result of Arhangel'skii [A2],

$$
H_{1}(K)=\mathrm{cl}_{A}\left(\bigcup\left\{\operatorname{supp}\left(r_{1}(x)\right): x \in n_{X}(K)\right\}\right)
$$

is a bounded subset of $A$. Thus, $H_{1}(K)$ is compact. So $H_{1}(K) \times n_{Y}(K)$ is a compact subset of $A \times Y$. Since $r(x, y)=\left(\operatorname{supp}\left(r_{1}(x)\right)\right) \times\{y\}$ for every point $(x, y) \in X \times Y$, we have $H(K) \subset H_{1}(K) \times n_{Y}(K)$. Hence, $H(K)$ is compact as a closed subset of $H_{1}(K) \times n_{Y}(K)$. Claim 2 is proved.

Now, the proof of Lemma 2.3 follows form the above two claims and the arguments used in the proof of Lemma 2.2.

2.4 Corollary. Let $X$ be a product of metric spaces and $A$ be an $l^{*}$-embedded subset of $X$. Then $\left(\prod C_{P}(X)\right)_{c} \sim\left(\prod C_{P}(A)\right)_{c} \times\left(\prod C_{P}(X ; A)\right)_{c}$.

Proof. Since $A$ is closed in $X$, every closed bounded subset of $A$ is compact. Thus, the proof follows from Lemma 2.3, where $Y$ is the one-point space.

2.5 Lemma. Suppose $X$ is a space such that both $X \times I$ and $X \times T$ are $k_{R^{-}}$ spaces, where $T=\{0,1 / n: n \in N\}$. Then $C_{P}(X \times I)$ is linearly homeomorphic to $\left(\prod C_{P}(X \times I)\right)_{c}$.

Proof. Since, by Lemma $2.1, X \times T$ is strongly $l$-embedded in $X \times I$ we have

$$
C_{P}(X \times I) \sim C_{P}(X \times T) \times C_{P}(X \times I ; X \times T) .
$$

Let $I_{n}=[1 / n+1,1 / n]$ and $E_{n}=C_{P}\left(X \times I_{n} ; X \times\{1 / n+1,1 / n\}\right)$ for every $n \in N$. Consider the set

$$
\begin{aligned}
& \left(\prod E_{n}\right)_{c}=\left\{\left(f_{1}, \ldots, f_{n}, \ldots\right) \in \prod E_{n}: \lim _{n}\left\|f_{n}\right\|_{K \times I_{n}}=0\right. \\
& \text { for every compact subset } K \text { of } X\}
\end{aligned}
$$

as a topological linear subset of $\prod\left\{E_{n}: n \in N\right\}$. Since $X \times I$ is a $k_{R}$-space 
we have $C_{P}(X \times I ; X \times T) \sim\left(\prod E_{n}\right)_{c}$. Identifying each $E_{n}$ with the space $E=C_{P}(X \times I ; X \times\{0,1\})$ we get

$$
C_{P}(X \times I ; X \times T) \sim\left(\prod E\right)_{c} .
$$

Analogously, $C_{P}(X \times T) \sim C_{P}(X \times\{0\}) \times C_{P}(X \times T ; X \times\{0\})$ and

$$
C_{P}(X \times T ; X \times\{0\}) \sim\left(\prod C_{P}(X)\right)_{c} .
$$

Thus,

$$
C_{P}(X \times T) \sim C_{P}(X \times\{0\}) \times\left(\prod C_{P}(X)\right)_{c} \sim\left(\prod C_{P}(X)\right)_{c} .
$$

By Lemma 2.3, the following holds

$$
\left(\prod C_{P}(X \times I)\right)_{c} \sim\left(\prod C_{P}(X \times\{0,1\})\right)_{c} \times\left(\prod E\right)_{c} .
$$

Obviously,

$$
\left(\prod C_{P}(X \times\{0,1\})\right)_{c} \sim\left(\prod C_{P}(X)\right)_{c} \times\left(\prod C_{P}(X)\right)_{c} \sim\left(\prod C_{P}(X)\right)_{c} .
$$

So we have

$$
\begin{aligned}
C_{P}(X \times I) & \sim C_{P}(X \times T) \times C_{P}(X \times I ; X \times T) \quad \text { by (1) } \\
& \sim\left(\prod C_{P}(X)\right)_{c} \times\left(\prod E\right)_{c} \quad \text { by }(2) \text { and }(3) \\
& \sim\left(\prod C_{P}(X \times I)\right)_{c} \quad \text { by }(4) \text { and }(5) .
\end{aligned}
$$

2.6 Corollary. Let $X$ be as in Lemma 2.5. Then $C_{P}(X \times I)$ is homeomorphic to $C_{P}(X \times I)^{\omega}$.

Proof. S. Gul'ko and T. Hmyleva [GH] proved that $\left(\prod C_{P}(X)\right)_{0}$ is homeomorphic to $C_{P}(X)^{\omega} \times\left(\prod C_{P}(X)\right)_{0}$ for every pseudocompact space $X$. Using the same arguments one can see that $\left(\prod C_{P}(X)\right)_{c}$ is homeomorphic to $C_{P}(X)^{\omega} \times\left(\prod C_{P}(X)\right)_{c}$ for each $X$. Now, the proof of Corollary 2.6 follows from Lemma 2.5 .

2.7 Lemma. Suppose a space $X$ contains an l-embedded copy $F_{1}$ of a space $Y$ and $Y$ contains an $l^{*}$-embedded copy $F_{2}$ of $X$. Then $C_{P}(X) \sim C_{P}(Y)$ provided one of the following conditions is fulfilled:

(i) $C_{P}(Y) \sim\left(\prod C_{P}(Y)\right)_{b}$ :

(ii) $C_{P}(Y) \sim\left(\prod C_{P}(Y)\right)_{c} \sim\left(\prod C_{P}\left(F_{2}\right)\right)_{c} \times\left(\prod C_{P}\left(Y ; F_{2}\right)\right)_{c}$.

Proof. We have $C_{P}(X) \sim C_{P}\left(F_{1}\right) \times E_{1}$ and $C_{P}(Y) \sim C_{P}\left(F_{2}\right) \times E_{2}$, where $E_{1}=C_{P}\left(X ; F_{1}\right)$ and $E_{2}=C_{P}\left(Y ; F_{2}\right)$. Thus, $C_{P}(X) \sim C_{P}(Y) \times E_{1}$. Suppose $C_{P}(Y) \sim\left(\prod C_{P}(Y)\right)_{b}$. By Lemma 2.2,

so

$$
\left(\prod C_{P}(Y)\right)_{b} \sim\left(\prod C_{P}\left(F_{2}\right)\right)_{b} \times\left(\prod E_{2}\right)_{b},
$$

$$
\left(\prod C_{P}(Y)\right)_{b} \sim\left(\prod C_{P}(X)\right)_{b} \times\left(\prod E_{2}\right)_{b}
$$


Therefore,

$$
\begin{aligned}
C_{P}(Y) & \sim\left(\prod C_{P}(Y)\right)_{b} \sim C_{P}(Y) \times\left(\prod C_{P}(Y)\right)_{b} \\
& \sim C_{P}(Y) \times\left(\prod C_{P}(X)\right)_{b} \times\left(\prod E_{2}\right)_{b} .
\end{aligned}
$$

Hence, $C_{P}(X) \sim E_{1} \times C_{P}(Y) \sim E_{1} \times C_{P}(Y) \times\left(\prod C_{P}(X)\right)_{b} \times\left(\prod E_{2}\right)_{b} \sim$ $C_{P}(X) \times\left(\prod C_{P}(X)\right)_{b} \times\left(\prod E_{2}\right)_{b} \sim\left(\prod C_{P}(X)\right)_{b} \times\left(\prod E_{2}\right)_{b} \sim C_{P}(Y)$.

If condition (ii) is fulfilled we use the same arguments.

2.8 Theorem. (i) Let $X$ be a subspace of $R^{n}$. Then $C_{P}(X) \sim C_{P}\left(I^{n}\right)$ if and only if $X$ is compact and $\operatorname{dim} X=n$;

(ii) $C_{P}(X) \sim C_{P}(Q)$ if and only if $X$ is a compact metric space containing a copy of $Q$.

Proof. We prove only the first part of Theorem 2.8. The proof of (ii) is analogous to that of $(\mathrm{i})$.

Suppose $C_{P}(X) \sim C_{P}\left(I^{n}\right)$. Then by [A2 and A3] $X$ is a compact metric space. Next, it follows from a result of Pavlovskii [Pv] that there is a nonempty open subset of $I^{n}$ which can be embedded in $X$. Thus, $\operatorname{dim} X=n$.

Now, let $X$ be a compact $n$-dimensional subset of $R^{n}$. Then $X$ contains a copy of $I^{n}$. On the other hand $X$ can be considered as a subset of $I^{n}$. Hence, by Corollary 2.4, $\left(\prod C_{P}\left(I^{n}\right)\right)_{c} \sim\left(\prod C_{P}(X)\right)_{c} \times\left(\prod C_{P}\left(I^{n} ; X\right)\right)_{c}$. Since $C_{P}\left(I^{n}\right) \sim\left(\prod C_{P}\left(I^{n}\right)\right)_{c}$ (see Lemma 2.5), we derive from Lemma 2.7 (ii) that $C_{P}(X) \sim C_{P}\left(I^{n}\right)$.

2.9 Theorem. Let $\mu^{n}$ be the n-dimensional universal Menger compactum. Then $C_{P}(X) \sim C_{P}\left(\mu^{n}\right)$ if and only if $X$ is an $n$-dimensional compact metric space containing a copy of $\mu^{n}$.

Proof. Let $C_{P}(X) \sim C_{P}\left(\mu^{n}\right)$. Then, by results of Arhangel'skii [A2, A3] and Pestov [Ps], $X$ is an $n$-dimensional compact metric space. It follows from [Pv] that there exists an open subset of $\mu^{n}$ which can be embedded in $X$. But each open subset of $\mu^{n}$ contains a copy of $\mu^{n}$ [Bt]. Thus, $X$ contains a copy of $\mu^{n}$.

Suppose $X$ is an $n$-dimensional compact metric space containing a copy of $\mu^{n}$. Since $X$ can be embedded in $\mu^{n}$, by Lemma 2.7(ii) and Corollary 2.4 it is enough to show that $C_{P}\left(\mu^{n}\right) \sim\left(\prod C_{P}\left(\mu^{n}\right)\right)_{c}$. For proving this fact we need the following result of Dranishnikov [Dr2]: There is a mapping $f_{n}$ from $\mu^{n}$ onto $Q$ such that $f_{n}^{-1}(P)$ is homeomorphic to $\mu^{n}$ for every $L C^{n-1} \& C^{n-1}$-compact subspace $P$ of $Q$. Now, consider $Q$ as a product $Q_{1} \times I$, where $Q_{1}$ is a copy of $Q$. Let $T=\{0,1 / k ; k \in N\}$ and $T^{*}=f_{n}^{-1}\left(Q_{1} \times T\right)$. Then

$$
C_{P}\left(\mu^{n}\right) \sim C_{P}\left(T^{*}\right) \times C_{P}\left(\mu^{n} ; T^{*}\right)
$$

and

$$
C_{P}\left(T^{*}\right) \sim C_{P}\left(f_{n}^{-1}\left(Q_{1} \times\{0\}\right)\right) \times C_{P}\left(T^{*} ; f_{n}^{-1}\left(Q_{1} \times\{0\}\right)\right) .
$$


Since each of the sets $f_{n}^{-1}\left(Q_{1} \times\{1 / k\}\right), k \in N$, and $f_{n}^{-1}\left(Q_{1} \times\{0\}\right)$ is homeomorphic to $\mu^{n}$, we have

$$
C_{P}\left(T^{*} ; f_{n}^{-1}\left(Q_{1} \times\{0\}\right)\right) \sim\left(\prod C_{P}\left(\mu^{n}\right)\right)_{c}
$$

and

$$
C_{P}\left(f_{n}^{-1}\left(Q_{1} \times\{0\}\right)\right) \sim C_{P}\left(\mu^{n}\right) .
$$

Thus,

$$
\begin{aligned}
C_{P}\left(T^{*}\right) & \sim C_{P}\left(\mu^{n}\right) \times\left(\prod C_{P}\left(\mu^{n}\right)\right)_{c} \sim\left(\prod C_{P}\left(\mu^{n}\right)\right)_{c} \\
& \sim\left(\prod C_{P}\left(\mu^{n}\right)\right)_{c} \times\left(\prod C_{P}\left(\mu^{n}\right)\right)_{c} \sim\left(\prod C_{P}\left(\mu^{n}\right)\right)_{c} \times C_{P}\left(T^{*}\right) .
\end{aligned}
$$

Finally,

$$
\begin{aligned}
C_{P}\left(\mu^{n}\right) & \sim C_{P}\left(T^{*}\right) \times C_{P}\left(\mu^{n} ; T^{*}\right) \quad \text { by }(6) \\
& \sim\left(\prod C_{P}\left(\mu^{n}\right)\right)_{c} \times C_{P}\left(T^{*}\right) \times C_{P}\left(\mu^{n} ; T^{*}\right) \text { by }(7) \\
& \sim\left(\prod C_{P}\left(\mu^{n}\right)\right)_{c} \times C_{P}\left(\mu^{n}\right) \sim\left(\prod C_{P}\left(\mu^{n}\right)\right)_{c} .
\end{aligned}
$$

2.10 Theorem. Let $X$ be a metric space and $\tau$ be an infinite cardinal. Suppose $Y$ is an $l^{*}$-embedded subspace of the product $X^{\tau}$ and $Y$ contains an $l^{*}$-embedded copy of $X^{\tau}$. Then $C_{P}(Y) \sim C_{P}\left(X^{\tau}\right)$.

Proof. By Corollary 2.4 and Lemma 2.7(ii), it is enough to show that $C_{P}\left(X^{\tau}\right) \sim$ $\left(\prod C_{P}\left(X^{\tau}\right)\right)_{c}$. Since $\tau$ is infinite we have $X^{\tau}=\left(X^{\omega}\right)^{\tau}$. So we can suppose that $X$ is not discrete. Thus, there exists a nontrivial converging sequence $\left\{x_{n}\right\}_{n \in N}$ in $X$ with $\lim x_{n}=x_{0}$. Let $T=\left\{x_{0}, x_{n} ; n \in N\right\}$. By Lemma $2.1, X^{\tau} \times T$ is $l$-embedded in $X^{\tau} \times X$. Therefore,

$$
C_{P}\left(X^{\tau}\right) \sim C_{P}\left(X^{\tau} \times T\right) \times C_{P}\left(X^{\tau} \times X ; X^{\tau} \times T\right) .
$$

But $C_{P}\left(X^{\tau} \times T\right) \sim C_{P}\left(X^{\tau} \times\left\{x_{0}\right\}\right) \times C_{P}\left(X^{\tau} \times T ; X^{\tau} \times\left\{x_{0}\right\}\right)$ because $X^{\tau} \times\left\{x_{0}\right\}$ is also l-embedded in $X^{\tau} \times T$. Since $X^{\tau} \times T$ is a $k_{R}$-space [N] we have $C_{P}\left(X^{\tau} \times T ; X^{\tau} \times\left\{x_{0}\right\}\right) \sim\left(\prod C_{P}\left(X^{\tau}\right)\right)_{c}$. Hence,

$$
\begin{aligned}
C_{P}\left(X^{\tau} \times T\right) & \sim C_{P}\left(X^{\tau} \times\left\{x_{0}\right\}\right) \times\left(\prod C_{P}\left(X^{\tau}\right)\right)_{c} \sim\left(\prod C_{P}\left(X^{\tau}\right)\right)_{c} \\
& \sim\left(\prod C_{P}\left(X^{\tau}\right)\right)_{c} \times\left(\prod C_{P}\left(X^{\tau}\right)\right)_{c} \sim C_{P}\left(X^{\tau} \times T\right) \times\left(\prod C_{P}\left(X^{\tau}\right)\right)_{c} .
\end{aligned}
$$

Then

$$
\begin{aligned}
C_{P}\left(X^{\tau}\right) & \sim C_{P}\left(X^{\tau} \times T\right) \times C_{P}\left(X^{\tau} \times X ; X^{\tau} \times T\right) \\
& \sim\left(\prod C_{P}\left(X^{\tau}\right)\right)_{c} \times C_{P}\left(X^{\tau} \times T\right) \times C_{P}\left(X^{\tau} \times X ; X^{\tau} \times T\right) \\
& \sim\left(\prod C_{P}\left(X^{\tau}\right)\right)_{c} \times C_{P}\left(X^{\tau}\right) \sim\left(\prod C_{P}\left(X^{\tau}\right)\right)_{c} .
\end{aligned}
$$


2.11 Corollary. Let $X$ be a separable metric space and $\tau>\omega$. Then $C_{P}\left(X^{\tau}\right)$ $\sim C_{P}(Y)$ for every closed $G_{\delta}$-subset $Y$ of $X^{\tau}$.

Proof. Suppose $Y$ is a closed $G_{\delta}$-subset of $X^{\tau}$. It is well known (see for example [PP]) that modulo a permutation of the coordinates, $Y=Z \times X^{\tau-\omega}$, where $Z$ is a closed subset of $X^{\omega}$. Thus, by Lemma $2.1, Y$ is $l^{*}$-embedded in $X^{\tau}$. On the other hand $\{z\} \times X^{\tau-\omega}$ is an $l^{*}$-embedded copy of $X^{\tau}$ in $Y$ for each $z \in Z$. Now, Theorem 2.10 completes the proof.

2.12 Corollary. Let $U$ be a functionally open subset of $R^{\tau}, \tau \geq \omega$. Then $C_{P}(U) \sim C_{P}\left(R^{\tau}\right)$.

Proof. Modulo a permutation of the coordinates, $U=V \times R^{\tau-\omega}$, where $V$ is open in $R^{\omega}$. Obviously, $U$ contains an $l^{*}$-embedded copy of $R^{\tau}$. Since there is an embedding of $V$ in $R^{\omega}$ as a closed subset, by Lemma 2.1, $U$ can be $l^{*}$-embedded in $R^{\tau}$. Thus, by Theorem 2.10, $C_{P}(U) \sim C_{P}\left(R^{\tau}\right)$.

Let $f$ be a mapping from a space $X$ onto a space $Y$. Recall that a continuous linear operator $u: C_{P}(X) \rightarrow C_{P}(Y)$ is said to be an averaging operator for $f$ if $u(h \circ f)=h$ for every $h \in C_{P}(Y)$. If $f$ admits a regular averaging operator $u: C_{P}(X) \rightarrow C_{P}(Y)$ we can define a mapping $r: Y \rightarrow P_{\infty}(X)$ by the formula $r(y)(g)=u(g)(y)$. The mapping $r$ has the following property [Dr1]: $\operatorname{supp}(r(y))$ is contained in $f^{-1}(y)$ for each $y \in Y$. Conversely, if there is a mapping $r: Y \rightarrow P_{\infty}(X)$ such that $\operatorname{supp}(r(y)) \subset f^{-1}(y)$ for every $y \in Y$, then the formula $u(g)(y)=r(y)(g)$ defines a regular averaging operator $u$ for $f$. It is easily seen that if $u$ is a regular averaging operator for $f$ the mapping $v(g)=(u(g), g-u(g) \circ f)$ is a linear homeomorphism from $C_{P}(X)$ onto $C_{P}(Y) \times E$, where $E=\left\{g-u(g) \circ f: g \in C_{P}(X)\right\}$. Dranishnikov proved [Dr1, Theorem 9] that $C_{P}\left(R^{n}\right) \sim C_{P}(U)$ for every open subset $U$ of $R^{n}$. The same arguments are used in the proof of Proposition 2.13 below.

2.13 Proposition. Let $\left\{U_{i}: i \in N\right\}$ be an infinite locally finite functionally open cover of a space $X$. Suppose there is a space $Y$ with $C_{P}\left(\mathrm{cl}_{X}\left(U_{i}\right)\right) \sim C_{P}(Y)$ for each $i \in N$. Then $C_{P}(X) \sim C_{P}(Y)^{\omega}$ provided $X$ contains an l-embedded copy of a topological sum $\sum_{i=1}^{\infty} F_{i}$ such that $C_{P}\left(F_{i}\right) \sim C_{P}(Y)$ for every $i \in N$.

Proof. For every $i \in N$ take an $f_{i} \in C_{P}(X)$ such that $f_{i}^{-1}(0)=X-U_{i}$ and $f_{i} \geq 0$. Without loss of generality we can suppose that $\sum_{i=1}^{\infty} f_{i}=1$. Let $f \in$ $C_{P}\left(\sum \mathrm{cl}_{X}\left(U_{i}\right)\right)$ such that $f\left|\mathrm{cl}_{X}\left(U_{i}\right)=f_{i}\right| \mathrm{cl}_{X}\left(U_{i}\right)$. Consider the natural mapping $p: \sum \mathrm{cl}_{X}\left(U_{i}\right) \rightarrow X$ with all preimages finite. Let $r: X \rightarrow P_{\infty}\left(\sum \mathrm{cl}_{X}\left(U_{i}\right)\right)$ be defined by $r(x)=\sum\left\{f(y) \cdot \delta_{y}: y \in p^{-1}(x)\right\}$. It is easily seen that $r$ is continuous and $\operatorname{supp}(r(x)) \subset p^{-1}(x)$ for every $x \in X$. Thus, there is a regular averaging operator $u: C_{P}\left(\sum \mathrm{cl}_{X}\left(U_{i}\right)\right) \rightarrow C_{P}(X)$ for $p$. Hence, $C_{P}\left(\sum \mathrm{cl}_{X}\left(U_{i}\right)\right)$ is linearly homeomorphic to $C_{P}(X) \times E$, where $E$ is a linear subspace of $C_{P}\left(\sum \mathrm{cl}_{X}\left(U_{i}\right)\right)$. Since $\sum F_{i}$ is $l$-embedded in $X$ we have $C_{P}(X) \sim C_{P}\left(\sum F_{i}\right) \times C_{P}\left(X ; \sum F_{i}\right)$. Observe that

$$
C_{P}\left(\sum \mathrm{cl}_{X}\left(U_{i}\right)\right) \sim \prod_{i=1}^{\infty} C_{P}\left(\mathrm{cl}_{X}\left(U_{i}\right)\right) \sim C_{P}(Y)^{\omega} \sim C_{P}\left(\sum F_{i}\right) .
$$


Now, using the technique of Pelczynski [P] and Bessaga [B] we have

$$
\begin{aligned}
C_{P}(X) & \sim C_{P}\left(\sum F_{i}\right) \times C_{P}\left(X ; \sum F_{i}\right) \sim C_{P}(Y)^{\omega} \times C_{P}\left(X ; \sum F_{i}\right) \\
& \sim\left(C_{P}(Y)^{\omega} \times \cdots \times C_{P}(Y)^{\omega} \times \cdots\right) \times C_{P}(Y)^{\omega} \times C_{P}\left(X ; \sum F_{i}\right) \\
& \sim\left(C_{P}(Y)^{\omega} \times \cdots \times C_{P}(Y)^{\omega} \times \cdots\right) \times C_{P}(X) \\
& \sim\left(C_{P}(X) \times E \times \cdots \times C_{P}(X) \times E \times \cdots\right) \times C_{P}(X) \\
& \sim C_{P}(X)^{\omega} \times E^{\omega} \sim\left(C_{P}(X) \times E\right)^{\omega} \sim C_{P}\left(\sum \operatorname{cl}_{X}\left(U_{i}\right)\right)^{\omega} \sim C_{P}(Y)^{\omega} .
\end{aligned}
$$

2.14 Theorem. Let $Y$ be a noncompact separable metric space and $X$ be one of the spaces $Q, I^{n}, \mu^{n}, l_{2}$. Then $C_{P}(Y) \sim C_{P}(X)^{\omega}$ provided $Y$ is an $X$ manifold.

Proof. Let $\left\{U_{i}: i \in N\right\}$ be an infinite locally finite open cover of $Y$ such that each $\mathrm{cl}_{Y}\left(U_{i}\right)$ is regularly closed subset of $X$. It is clear that a topological sum $\sum F_{i}$ of infinitely many regularly closed subsets $F_{i}$ of $X$ is contained in $Y$ as a closed subset. Since each of the sets $\mathrm{cl}_{Y}\left(U_{i}\right)$ and $F_{i}, i \in N$, contains a closed copy of $X$, it follows from Theorem 2.8, Theorem 2.9 and Theorem 2.10 that $C_{P}\left(\operatorname{cl}_{Y}\left(U_{i}\right)\right) \sim C_{P}\left(F_{i}\right) \sim C_{P}(X)$ for every $i \in N$. Hence, by Proposition 2.13, $C_{P}(Y) \sim C_{P}(X)^{\omega}$.

2.15 Theorem. Let $U$ be a functionally open subset of $I^{\tau}$ and $\tau$ be an uncountable cardinal. Then $C_{P}(U) \sim C_{P}\left(I^{\tau}\right)^{\omega}$.

Proof. There exists a projection $p$ from $I^{\tau}$ onto a countable face of $I^{\tau}$ such that $p^{-1}(p(U))=U$ (see [PP]). Take a locally finite open cover $\left\{U_{i}: i \in N\right\}$ of $p(U)$ such that $\mathrm{cl}_{I^{\tau}}\left(p^{-1}\left(U_{i}\right)\right) \subset U$ for every $i \in N$. Since each $\operatorname{cl}_{I^{\tau}}\left(p^{-1}\left(U_{i}\right)\right)$ is a closed $G_{\delta}$-subset of $I^{\tau}$, by Corollary 2.11, $C_{P}\left(\operatorname{cl}_{I^{\tau}}\left(p^{-1}\left(U_{i}\right)\right)\right) \sim C_{P}\left(I^{\tau}\right)$.

Now, let $\left\{x_{i}: i \in N\right\}$ be a closed discrete infinite subset of $p(U)$. So, the topological sum $\sum p^{-1}\left(x_{i}\right)$ is $l$-embedded in $U$ (by Lemma 2.1) and obviously, each $p^{-1}\left(x_{i}\right)$ is homeomorphic to $I^{\tau}$. Thus, by Proposition 2.13, $C_{P}(U) \sim$ $C_{P}\left(I^{\tau}\right)^{\omega}$.

2.16 Theorem. Let $X$ be one of the spaces $Q, I^{n}, \mu^{n}$, and $Y$ be a locally compact subset of an $X$-manifold. Then $C_{P}(Y) \sim C_{P}(X)^{\omega}$ if and only if $Y$ contains a closed copy of the topological sum $\sum X$ of infinitely many copies of $X$.

Proof. The proof of the part "if" is based on a Dranishnikov's idea from [Dr1, Theorem $9^{\prime}$ ], where it is shown that $C_{P}(P) \sim C_{P}\left(R^{n}\right)$ for every locally compact subset $P$ of $R^{n}$ with $\operatorname{cl}_{R^{n}}(\operatorname{Int}(P)) \cap\left(R^{n}-P\right) \neq \varnothing$.

Suppose $Y$ is a locally compact subspace of an $X$-manifold $Z$ and contains a closed copy of the topological sum $\sum X$. Then $C_{P}(Y) \sim C_{P}\left(\sum X\right) \times$ $C_{P}\left(Y ; \sum X\right)$. Next, take a locally finite open cover $\left\{V_{i}: i \in N\right\}$ of $Y$ such that each $\operatorname{cl}_{Y}\left(V_{i}\right)$ is compact. For every $i \in N$ there exists an open subset $U_{i}$ 
of $Z$ such that $V_{i}=U_{i} \cap Y=U_{i} \cap \mathrm{cl}_{Y}\left(V_{i}\right)$. Since every set $V_{i}$ is closed in $U_{i}, \sum V_{i}$ is closed in $\sum U_{i}$. Thus, $C_{P}\left(\sum U_{i}\right) \sim C_{P}\left(\sum V_{i}\right) \times C_{P}\left(\sum U_{i} ; \sum V_{i}\right)$. Let $\left\{f_{i}: i \in N\right\}$ be a partition of unity subordinated to the cover $\left\{V_{i}: i \in N\right\}$. Define a continuous mapping $r: Y \rightarrow P_{\infty}\left(\sum V_{i}\right)$ as in the proof of Proposition 2.13 and by the same arguments we get that $C_{P}\left(\sum V_{i}\right)$ is linearly homeomorphic to $C_{P}(Y) \times E$, where $E$ is a linear subspace of $C_{P}\left(\sum V_{i}\right)$. It follows from Theorem 2.14 that $C_{P}\left(U_{i}\right) \sim C_{P}(X)^{\omega}$ for every $i \in N$. Hence

$$
\begin{aligned}
C_{P}(X)^{\omega} & \sim C_{P}\left(\sum U_{i}\right) \sim C_{P}\left(\sum V_{i}\right) \times C_{P}\left(\sum U_{i} ; \sum V_{i}\right) \\
& \sim C_{P}(Y) \times E \times C_{P}\left(\sum U_{i} ; \sum V_{i}\right) .
\end{aligned}
$$

Now, using the scheme of Pelczynski and Bessaga we get $C_{P}(Y) \sim C_{P}(X)^{\omega}$.

Suppose there is a linear homeomorphism $\theta$ from $C_{P}\left(\sum X\right)=C_{P}(X)^{\omega}$ onto $C_{P}(Y)$. Let $K$ be the set $\{y \in Y$; every neighborhood of $y$ in $Y$ contains a copy of $X$ \}. We use the following property of $X$ (for $Q$ and $I^{n}$ this is obvious, and for $\mu^{n}$ see $[\mathrm{Bt}]$ ):

(*) Every open subset of $X$ contains a copy of $X$.

Now we show that $K$ is nonempty. Indeed, by [Pv], $Y$ contains an open subset of $\sum X$. So, by $(*), Y$ contains a copy $F$ of $X$ and $F \subset K$. Obviously $K$ is closed in $Y$ and it follows also from (*) that $Y-K$ does not contain a copy of $X$. Next, assume $K$ is compact. Consider the set

$$
L=\operatorname{cl}\left(\bigcup\left\{\operatorname{supp}\left(\theta^{*}\left(\delta_{y}\right)\right): y \in K\right\}\right),
$$

where $\theta^{*}: L_{P}(Y) \rightarrow L_{P}\left(\sum X\right)$ is the dual homeomorphism of $\theta$. By a result of Arhangel'skii [A2], $L$ is a compact subset of $\sum X$. Therefore, there is a $k \in N$ such that $L \subset \sum_{i=1}^{k} X_{i}$. Let $P=\sum_{i=1}^{k} X_{i}, f \in C_{P}\left(\sum X ; P\right)$ and $y \in K$. We have $\theta^{*}\left(\delta_{y}\right)(f)=\delta_{y}(\theta(f))=\theta(f)(y)$. But $\theta^{*}\left(\delta_{y}\right)(f)=$ 0 because $\operatorname{supp}\left(\theta^{*}\left(\delta_{y}\right)\right) \subset P$. Thus, $\theta(f)$ belongs to $C_{P}(Y ; K)$ for every $f \in C_{P}\left(\sum X ; P\right)$. Let $p$ be the linear projection from $C_{P}\left(\sum X\right)=C_{P}(P) \times$ $C_{P}\left(\sum X ; P\right)$ onto $C_{P}\left(\sum X ; P\right)$. Then $\theta \circ p \circ \theta^{-1}: C_{p}(Y ; K) \rightarrow \theta\left(C_{P}\left(\sum X ; P\right)\right)$ is a continuous linear retraction. This means that there is a closed linear subspace $E$ of $C_{P}(Y ; K)$ such that $C_{P}(Y ; K)$ is linearly homeomorphic to $C_{P}\left(\sum X ; P\right) \times E$. Clearly, $C_{P}(Y ; K) \sim C_{P}(Y / K ;(K))$, where $(K)$ is the identification point of $K$ in the quotient space $Y / K$. Analogously, $C_{P}\left(\sum X ; P\right) \sim$ $C_{P}\left(\left(\sum X\right) / P ;(P)\right)$. Since $C_{P}(Y / K) \sim R \times C_{P}(Y / K ;(K))$ and

$$
C_{P}\left(\left(\sum X\right) / P ;(P)\right) \times R \sim C_{P}\left(\left(\sum X\right) / P\right),
$$

we get that $C_{P}(Y / K) \sim C_{P}\left(\left(\sum X\right) / P\right) \times E$. Now, we need the following result of Dranishnikov [Drl, Theorem 6]: Let $X_{1}$ and $X_{2}$ be compact metric spaces and $C_{P}\left(X_{1}\right)$ be linearly homeomorphic to a product $C_{P}\left(X_{2}\right) \times E_{1}$. Then $\operatorname{dim} X_{2} \leq$ $\operatorname{dim} X_{1}$. Actually, it is proved that $X_{2}$ is a union of countably many compact subsets which are embeddable in $X_{1}$. It follows from Dranishnikov's arguments that the last statement remains valid if $X_{1}$ and $X_{2}$ are separable locally compact 
metric spaces. Hence, there is a countable family $\left\{F_{i}: i \in N\right\}$ of compact subsets of $\left(\sum X\right) / P$ such that $\left(\sum X\right) / P=\bigcup\left\{F_{i}: i \in N\right\}$ and each $F_{i}$ can be embedded in $Y / K$. Since $\left(\sum X\right) / P$ has the Baire property, there exists an $i_{0} \in N$ with $\operatorname{Int}\left(F_{i_{0}}\right) \neq \varnothing$. Then the set $\operatorname{Int}\left(F_{i_{0}}\right)-\{(P)\}$ is both open in $\sum X$ and embeddable in $Y / K$. Thus, by $(*), Y / K$ contains a copy of $X$. So $Y-K$ contains also a copy of $X$. But we have already seen that this is not possible. Therefore $K$ is not compact.

Take a countable infinite discrete family $\left\{W_{i}: i \in N\right\}$ in $K$ consisting of open subsets of $K$. Let $W_{i}^{*}$ be an open subspace of $Y$ with $W_{i}^{*} \cap K=W_{i}$ for each $i \in N$. For every $i \in N$ there is a copy $X_{i}$ of $X$ such that $X_{i} \subset W_{i}^{*}$. It follows from (*) that $X_{i} \subset K$ because $Y-K$ does not contain a copy of $X$. Hence, $X_{i} \subset W_{i}$ for every $i \in N$. So $\left\{X_{i}: i \in N\right\}$ is a discrete family in $K$. Thus, $\sum X_{i}$ is a closed subset of $Y$.

2.17 Corollary. Let $X$ be a locally compact ( $n$-dimensional) separable metric space. Then $C_{P}(X) \sim C_{P}(Q)^{\omega}$ (resp., $\left.C_{P}(X) \sim C_{P}\left(\mu^{n}\right)^{\omega}\right)$ if and only if $X$ contains a closed copy of the topological sum $\sum Q$ (resp., $\left.\sum \mu^{n}\right)$.

Proof. Since $X$ can be embedded in $Q$ (resp., in $\mu^{n}$ ), the proof follows from Theorem 2.16.

\section{LiNEAR TOPOLOGICAL CLASSIFICATIONS OF $C_{P}^{*}(X)$}

The proofs of the Lemmas 3.1-3.4 below are similar to the proofs of the corresponding lemmas from $\S 2$.

3.1 Lemma. Let $A$ be an $l^{*}$-embedded subset of a space $X$. Then $\left(\prod C_{P}^{*}(X)\right)_{b}^{*}$ $\sim\left(\prod C_{P}^{*}(A)\right)_{b}^{*} \times\left(\prod C_{P}^{*}(X ; A)\right)_{b}^{*}$.

3.2 Lemma. Let $A$ be an $l^{*}$-embedded subset of a space $X$. If every closed bounded subset of $A$ is compact then $\left(\prod C_{P}^{*}(X \times Y)\right)_{c}^{*} \sim\left(\prod C_{P}^{*}(A \times Y)\right)_{c}^{*} \times$ $\left(\prod C_{P}^{*}(X \times Y ; A \times Y)\right)_{c}^{*}$ for any space $Y$.

3.3 Corollary. Let $A$ be an $l^{*}$-embedded subset of a product $X$ of metric spaces. Then

$$
\left(\prod C_{P}^{*}(X)\right)_{c}^{*} \sim\left(\prod C_{P}^{*}(A)\right)_{c}^{*} \times\left(\prod C_{P}^{*}(X ; A)\right)_{c}^{*}
$$

3.4 Lemma. Suppose $X$ is a space such that both $X \times T$ and $X \times I$ are $k_{R^{-}}$ spaces, where $T=\{0,1 / n: n \in N\}$. Then we have $C_{P}^{*}(X \times I) \sim\left(\prod C_{P}^{*}(X \times I)\right)_{c}^{*}$.

3.5 Corollary. Let $X=\sum I^{\tau}$ be a topological sum of infinitely many copies of $I^{\tau}, \tau \geq 1$. Then $C_{P}^{*}(X) \sim\left(\prod C_{P}^{*}(X)\right)_{c}^{*}$.

3.6 Lemma. Suppose a space $X$ contains an $l^{*}$-embedded copy $F_{1}$ of a space $Y$ and $Y$ contains an $l^{*}$-embedded copy $F_{2}$ of $X$. Then:

(i) $C_{P}^{*}(X) \sim\left(\prod C_{P}^{*}(X)\right)_{b}^{*} \sim C_{P}^{*}(Y)$ if $C_{P}^{*}(Y) \sim\left(\prod C_{P}^{*}(Y)\right)_{b}^{*}$;

(ii) $C_{P}^{*}(X) \sim\left(\prod C_{P}^{*}(X)\right)_{c}^{*} \sim C_{P}^{*}(Y)$ if $C_{P}^{*}(Y) \sim\left(\prod C_{P}^{*}(Y)\right)_{c}^{*} \sim\left(\prod C_{P}^{*}\left(F_{2}\right)\right)_{c}^{*}$ $\times\left(\prod C_{P}^{*}\left(Y ; F_{2}\right)\right)_{C}^{*}$. 
Proof. Let $C_{P}^{*}(Y) \sim\left(\prod C_{P}^{*}(Y)\right)_{b}^{*}$. Using the same arguments as in the proof of Lemma $2.7(\mathrm{i})$, one can show that $C_{P}^{*}(X) \sim C_{P}^{*}(Y)$. Next, by Lemma 3.1 , we have

$$
\left(\prod C_{P}^{*}(X)\right)_{b}^{*} \sim\left(\prod C_{P}^{*}\left(F_{1}\right)\right)_{b}^{*} \times\left(\prod C_{P}^{*}\left(X ; F_{1}\right)\right)_{b}^{*}
$$

and

$$
\left(\prod C_{P}^{*}(Y)\right)_{b}^{*} \sim\left(\prod C_{P}^{*}\left(F_{2}\right)\right)_{b}^{*} \times\left(\prod C_{P}^{*}\left(Y ; F_{2}\right)\right)_{b}^{*}
$$

Thus,

$$
\begin{aligned}
\left(\prod C_{P}^{*}(X)\right)_{b}^{*} & \sim\left(\prod C_{P}^{*}\left(F_{1}\right)\right)_{b}^{*} \times\left(\prod C_{P}^{*}\left(X ; F_{1}\right)\right)_{b}^{*} \\
& \sim\left(\prod C_{P}^{*}\left(F_{1}\right)\right)_{b}^{*} \times\left(\prod C_{P}^{*}\left(F_{1}\right)\right)_{b}^{*} \times\left(\prod C_{P}^{*}\left(X ; F_{1}\right)\right)_{b}^{*} \\
& \sim\left(\prod C_{P}^{*}\left(F_{1}\right)\right)_{b}^{*} \times\left(\prod C_{P}^{*}(X)\right)_{b}^{*} \\
& \sim\left(\prod C_{P}^{*}(Y)\right)_{b}^{*} \times\left(\prod C_{P}^{*}(X)\right)_{b}^{*} \\
& \sim\left(\prod C_{P}^{*}\left(F_{2}\right)\right)_{b}^{*} \times\left(\prod C_{P}^{*}\left(Y ; F_{2}\right)\right)_{b}^{*} \times\left(\prod C_{i}^{*}(X)\right)_{b}^{*} \\
& \sim\left(\prod C_{P}^{*}\left(F_{2}\right)\right)_{b}^{*} \times\left(\prod C_{P}^{*}\left(Y ; F_{2}\right)\right)_{b}^{*} \times\left(\prod C_{P}^{*}\left(F_{2}\right)\right)_{b}^{*} \\
& \sim\left(\prod C_{P}^{*}\left(F_{2}\right)\right)_{b}^{*} \times\left(\prod C_{P}^{*}\left(Y ; F_{2}\right)\right)_{b}^{*} \\
& \sim\left(\prod C_{P}^{*}(Y)\right)_{b}^{*} \sim C_{P}^{*}(Y) \sim C_{P}^{*}(X) .
\end{aligned}
$$

Using the same arguments we can prove that $\left(\prod C_{P}^{*}(X)\right)_{c}^{*} \sim C_{P}^{*}(X) \sim C_{P}^{*}(Y)$ if $C_{P}^{*}(Y) \sim\left(\prod C_{P}^{*}\left(F_{2}\right)\right)_{c}^{*} \times\left(\prod C_{P}^{*}\left(Y ; F_{2}\right)\right)_{c}^{*} \sim\left(\prod C_{P}^{*}(Y)\right)_{c}^{*}$.

3.7 Corollary. Let $\left\{X_{i}: i \in N\right\}$ be an infinite family of spaces such that each $X_{i}$ is strongly l-embedded in a space $Y$ and contains a strongly l-embedded copy $Y_{i}$ of $Y$. Then $C_{P}^{*}\left(\sum Y_{i}\right) \sim\left(\prod C_{P}^{*}\left(\sum X_{i}\right)\right)_{b}^{*} \sim C_{P}^{*}\left(\sum X_{i}\right)$ if $C_{P}^{*}\left(\sum Y_{i}\right) \sim$ $\left(\prod C_{P}^{*}\left(\sum Y_{i}\right)\right)_{b}^{*}$.

Proof. Let for each $i \quad u_{i}: C_{P}\left(X_{i}\right) \rightarrow C_{P}(Y)$ be a regular extension operator. Then the mapping $u: C_{P}\left(\sum X_{i}\right) \rightarrow C_{P}\left(\sum Y_{i}\right)$, defined by $u(f)=\sum u_{i}\left(f \mid X_{i}\right)$ is also a regular extension operator. Thus, $\sum X_{i}$ is $l^{*}$-embedded in $\sum Y_{i}$. Analogously, $\sum Y_{i}$ is $l^{*}$-embedded in $\sum X_{i}$. Now the proof follows from Lemma 3.6(i).

3.8 Theorem. Let $X$ be a metric space and $\tau$ be an infinite cardinal. Suppose $Y$ is an $l^{*}$-embedded subspace of the product $X^{\tau}$ and $Y$ contains an $l^{*}$-embedded copy of $X^{\tau}$. Then $C_{P}^{*}(Y) \sim C_{P}^{*}\left(X^{\tau}\right) \sim\left(\prod C_{P}^{*}\left(X^{\tau}\right)\right)_{c}^{*}$.

Proof. By Corollary 3.3 and Lemma 3.6(ii), it is enough to show that $C_{P}^{*}\left(X^{\tau}\right) \sim$ $\left(\prod C_{P}^{*}\left(X^{\tau}\right)\right)_{c}^{*}$. The last can be proved using the same arguments as in the proof of Theorem 2.10 .

3.9 Corollary. Let $X$ be a separable metric space and $\tau>\omega$. Then $C_{P}^{*}\left(X^{\tau}\right) \sim$ $C_{P}^{*}(Y)$ for every closed $G_{\delta}$-subset $Y$ of $X^{\tau}$. 
3.10 Corollary. Let $U$ be a functionally open subset of $R^{\tau}, \tau \geq \omega$. Then $C_{P}^{*}\left(R^{\tau}\right) \sim C_{P}^{*}(U)$.

The proofs of Corollaries 3.9 and 3.10 are similar respectively to the proofs of Corollaries 2.11 and 2.12 .

3.11 Proposition. Let $\sum \mu_{i}^{n}$ be a topological sum of infinitely many copies of the n-dimensional Menger compactum. Then $C_{P}^{*}\left(\sum \mu_{i}^{n}\right) \sim\left(\prod C_{P}^{*}\left(\sum \mu_{i}^{n}\right)\right)_{c}^{*}$.

Proof. For each $i \in N$ take a mapping $f_{n}^{i}$ from $\mu_{i}^{n}$ onto a copy $Q_{i}$ of the Hilbert cube $Q$ such that $\left(f_{n}^{i}\right)^{-1}(P)$ is homeomorphic to $\mu^{n}$ for every $L C^{n-1} \& C^{n-1}$-compact subspace $P$ of $Q_{i}$ (see [Dr2]). Define $f_{n}: \sum \mu_{i}^{n} \rightarrow \sum Q_{i}$ by $f_{n} \mid \mu_{i}^{n}=f_{n}^{i}$. Consider $Q_{i}$ as a product $Q_{i}^{1} \times I$, where $Q_{i}^{1}$ is a copy of $Q$. Let $T_{i}=Q_{i}^{1} \times\{0,1 / k: k \in N\}$ and $T=f_{n}^{-1}\left(\sum T_{i}\right)$. Then we have

$$
C_{P}^{*}\left(\sum \mu_{i}^{n}\right) \sim C_{P}^{*}(T) \times C_{P}^{*}\left(\sum \mu_{i}^{n} ; T\right)
$$

and

$$
C_{P}^{*}(T) \sim C_{P}^{*}\left(f_{n}^{-1}\left(\sum\left(Q_{i}^{1} \times\{0\}\right)\right)\right) \times C_{P}^{*}\left(T ; f_{n}^{-1}\left(\sum\left(Q_{i}^{1} \times\{0\}\right)\right)\right) .
$$

Since each of the sets $f_{n}^{-1}\left(\sum\left(Q_{i}^{1} \times\{0\}\right)\right)$ and $f_{n}^{-1}\left(\sum\left(Q_{i}^{1} \times\{1 / k\}\right)\right)$ for $k \in N$ is homeomorphic to $\sum \mu_{i}^{n}$, the following holds

$$
C_{P}^{*}\left(f_{n}^{-1}\left(\sum\left(Q_{i}^{1} \times\{0\}\right)\right)\right) \sim C_{P}^{*}\left(\sum \mu_{i}^{n}\right)
$$

and

$$
C_{P}^{*}\left(T ; f_{n}^{-1}\left(\sum\left(Q_{i}^{1} \times\{0\}\right)\right)\right) \sim\left(\prod C_{P}^{*}\left(\sum \mu_{i}^{n}\right)\right)_{c}^{*} .
$$

Thus,

$$
\begin{aligned}
C_{P}^{*}(T) & \sim C_{P}^{*}\left(\sum \mu_{i}^{n}\right) \times\left(\prod C_{P}^{*}\left(\sum \mu_{i}^{n}\right)\right)_{c}^{*} \sim\left(\prod C_{P}^{*}\left(\sum \mu_{i}^{n}\right)\right)_{c}^{*} \\
& \sim\left(\prod C_{P}^{*}\left(\sum \mu_{i}^{n}\right)\right)_{c}^{*} \times\left(\prod C_{P}^{*}\left(\sum \mu_{i}^{n}\right)\right)_{c}^{*} \\
& \sim\left(\prod C_{P}^{*}\left(\sum \mu_{i}^{n}\right)\right)_{c}^{*} \times C_{P}^{*}(T) .
\end{aligned}
$$

Finally we get

$$
\begin{aligned}
C_{P}^{*}\left(\sum \mu_{i}^{n}\right) & \sim C_{P}^{*}(T) \times C_{P}^{*}\left(\sum \mu_{i}^{n} ; T\right) \\
& \sim\left(\prod C_{P}^{*}\left(\sum \mu_{i}^{n}\right)\right)_{c}^{*} \times C_{P}^{*}(T) \times C_{P}^{*}\left(\sum \mu_{i}^{n} ; T\right) \\
& \sim\left(\prod C_{P}^{*}\left(\sum \mu_{i}^{n}\right)\right)_{c}^{*} \times C_{P}^{*}\left(\sum \mu_{i}^{n}\right) \sim\left(\prod C_{P}^{*}\left(\sum \mu_{i}^{n}\right)\right)_{c}^{*} .
\end{aligned}
$$

3.12 Lemma. Suppose $p$ is a mapping from a space $X$ onto a space $Y$ such that for every compact subset $K$ of $Y$ the preimage $p^{-1}(K)$ is also compact. 
Let $p$ admit a regular averaging operator $u: C_{p}(X) \rightarrow C_{p}(Y)$. Then $C_{p}^{*}(X) \sim$ $C_{p}^{*}(Y) \times E_{1}$ and $\left(\prod C_{p}^{*}(X)\right)_{c}^{*} \sim\left(\prod C_{p}^{*}(Y)\right)_{c}^{*} \times\left(\prod E_{1}\right)_{c}^{*}$, where $E_{1}=\{g-u(g) \circ$ $\left.p: g \in C_{p}^{*}(X)\right\}$.

Proof. Consider the mapping $r: Y \rightarrow P_{\infty}(X)$ defined by $r(y)(g)=u(g)(y)$ for all $g \in C_{P}(X)$. We have $\operatorname{supp}(r(y)) \subset p^{-1}(y)$ for each $y \in Y$. The last implies that $\|u(g)\|_{K} \leq\|g\|_{p^{-1}(K)}$ for every $g \in C_{P}^{*}(X)$ and $K \subset Y$. Hence, $u\left(C_{P}^{*}(X)\right)=C_{P}^{*}(Y)$ and the mapping $v(g)=(u(g), g-u(g) \circ p)$ is a linear homeomorphism from $C_{P}^{*}(X)$ onto $C_{P}^{*}(Y) \times E_{1}$. Next, let $\left(g_{1}, \ldots, g_{n}, \ldots\right) \in$ $\left(\Pi C_{P}^{*}(X)\right)_{c}^{*}$ and $K$ be a compact subset of $Y$. Since, $\left\|u\left(g_{n}\right)\right\|_{K} \leq\left\|g_{n}\right\|_{p^{-1}(K)}$ and $p^{-1}(K)$ is compact, we have $\left(u\left(g_{1}\right), \ldots, u\left(g_{n}\right), \ldots\right) \in\left(\prod C_{P}^{*}(Y)\right)_{c}^{*}$ and $\left(g_{1}-u\left(g_{1}\right) \circ p, \ldots, g_{n}-u\left(g_{n}\right) \circ p, \ldots\right) \in\left(\prod E_{1}\right)_{c}^{*}$. Obviously, $\left(g_{1}, \ldots, g_{n}, \ldots\right)$ $\in\left(\prod C_{P}^{*}(X)\right)_{c}^{*}$ if $\left(u\left(g_{1}\right), \ldots, u\left(g_{n}\right), \ldots\right) \in\left(\prod C_{P}^{*}(Y)\right)_{c}^{*}$ and $\left(g_{1}-u\left(g_{1}\right) \circ p\right.$, $\left.\ldots, g_{n}-u\left(g_{n}\right) \circ p, \ldots\right) \in\left(\prod E_{1}\right)_{c}^{*}$. Thus, the mapping

$$
\begin{aligned}
& v_{0}\left(g_{1}, \ldots, g_{n}, \ldots\right) \\
& \quad=\left(\left(u\left(g_{1}\right), \ldots, u\left(g_{n}\right), \ldots\right),\left(g_{1}-u\left(g_{1}\right) \circ p, \ldots, g_{n}-u\left(g_{n}\right) \circ p, \ldots\right)\right)
\end{aligned}
$$

is a linear homeomorphism from $\left(\prod C_{P}^{*}(X)\right)_{c}^{*}$ onto $\left(\prod C_{P}^{*}(Y)\right)_{c}^{*} \times\left(\prod E_{1}\right)_{c}^{*}$.

3.13 Proposition. Let $\left\{U_{i}: i \in N\right\}$ be an infinite locally finite functionally open cover of a space $X$. Suppose there is a space $Y$ such that $C_{P}^{*}(Y) \sim$ $C_{P}^{*}\left(\sum \operatorname{cl}_{X}\left(U_{i}\right)\right) \sim\left(\prod C_{P}^{*}\left(\sum \mathrm{cl}_{X}\left(U_{i}\right)\right)\right)_{c}^{*}$. Then $C_{P}^{*}(X) \sim C_{P}^{*}(Y)$ if $X$ contains an $l^{*}$-embedded copy of $Y$.

Proof. There exists a natural mapping $p$ from $\sum \mathrm{cl}_{X}\left(U_{i}\right)$ onto $X$ such that $p^{-1}(K)$ is compact for every compact subset $K$ of $X$. As in the proof of Proposition 2.13 we conclude that $p$ admits a regular averaging operator

$$
u: C_{P}\left(\sum \mathrm{cl}_{X}\left(U_{i}\right)\right) \rightarrow C_{P}(X) .
$$

By Lemma 3.12, $\left(\prod C_{P}^{*}\left(\sum \mathrm{cl}_{X}\left(U_{i}\right)\right)\right)_{c}^{*} \sim\left(\prod C_{P}^{*}(X)\right)_{c}^{*} \times\left(\prod E_{1}\right)_{c}^{*}$, where $E_{1}=$ $\left\{g-u(g) \circ p: g \in C_{P}^{*}\left(\sum \operatorname{cl}_{X}\left(U_{i}\right)\right)\right\}$. Since $Y$ is $l^{*}$-embedded in $X, C_{P}^{*}(X) \sim$ $C_{P}^{*}(Y) \times C_{P}^{*}(X ; Y)$. Then we have

$$
\begin{aligned}
C_{P}^{*}(X) & \sim C_{P}^{*}(Y) \times C_{P}^{*}(X ; Y) \sim\left(\prod C_{P}^{*}\left(\sum \mathrm{cl}_{X}\left(U_{i}\right)\right)\right)_{c}^{*} \times C_{P}^{*}(X ; Y) \\
& \sim\left(\prod C_{P}^{*}\left(\sum \mathrm{cl}_{X}\left(U_{i}\right)\right)\right)_{c}^{*} \times C_{P}^{*}\left(\sum \mathrm{cl}_{X}\left(U_{i}\right)\right) \times C_{P}^{*}(X ; Y) \\
& \sim\left(\prod C_{P}^{*}\left(\sum \mathrm{cl}_{X}\left(U_{i}\right)\right)\right)_{c}^{*} \times C_{P}^{*}(Y) \times C_{P}^{*}(X ; Y) \\
& \sim\left(\prod C_{P}^{*}\left(\sum \mathrm{cl}_{X}\left(U_{i}\right)\right)\right)_{c}^{*} \times C_{P}^{*}(X) \\
& \sim\left(\prod C_{P}^{*}(X)\right)_{c}^{*} \times\left(\prod E_{1}\right)_{c}^{*} \times C_{P}^{*}(X) \sim\left(\prod C_{P}^{*}(X)\right)_{c}^{*} \times\left(\prod E_{1}\right)_{c}^{*} \\
& \sim\left(\prod C_{P}^{*}\left(\sum \mathrm{cl}_{X}\left(U_{i}\right)\right)\right)_{c}^{*} \sim C_{P}^{*}(Y) .
\end{aligned}
$$


3.14 Theorem. Suppose $X$ is a noncompact $Y$-manifold, where $Y$ is one of the spaces $Q, I^{n}, \mu^{n}, l_{2}$. Then $C_{P}^{*}(X) \sim C_{P}^{*}\left(\sum Y\right)$.

Proof. Let $\left\{U_{i} ; i \in N\right\}$ be an infinite locally finite open cover of $X$ such that each $\mathrm{cl}_{X}\left(U_{i}\right)$ is regularly closed subset of $Y$. By Corollary 3.5, Proposition 3.11 and Theorem 3.8 we have $C_{P}^{*}\left(\sum Y\right) \sim\left(\prod C_{P}^{*}\left(\sum Y\right)\right)_{c}^{*}$. Since each set $\operatorname{cl}_{X}\left(U_{i}\right)$ is closed in $Y$ and contains a closed copy of $Y$, it follows from Corollary 3.7 that $\left(\prod C_{P}^{*}\left(\sum \mathrm{cl}_{X}\left(U_{i}\right)\right)\right)_{c}^{*} \sim C_{P}^{*}\left(\sum Y\right)$. Obviously $X$ contains a closed copy of $\sum Y$. Thus, by Proposition 3.13, $C_{P}^{*}(X) \sim C_{P}^{*}\left(\sum Y\right)$.

3.15 Theorem. Let $U$ be a functionally open subset of $I^{\tau}$ and $\tau$ be an uncountable cardinal. Then $C_{P}^{*}(U) \sim C_{P}^{*}\left(\sum I^{\tau}\right)$.

Proof. Take a projection $p$ from $I^{\tau}$ onto a countable face $I^{\omega}$ of $I^{\tau}$ such that $p^{-1}(p(U))=U$ (for the existence of a such projection see [PP]). Now, let $\left\{U_{i} ; i \in N\right\}$ be a locally finite open cover of $p(U)$ such that $\operatorname{cl}_{I^{\omega}}\left(U_{i}\right) \subset p(U)$ for each $i \in N$. Then $\left\{p^{-1}\left(U_{i}\right): i \in N\right\}$ is an infinite locally finite functionally open cover of $U$ with $\operatorname{cl}_{I^{\tau}}\left(p^{-1}\left(U_{i}\right)\right) \subset U$ for every $i \in N$. Since $p$ is an open mapping we have $\operatorname{cl}_{I^{\tau}}\left(p^{-1}\left(U_{i}\right)\right)=p^{-1}\left(\mathrm{cl}_{I^{\omega}}\left(U_{i}\right)\right)$. Thus, by Lemma 2.1, each set $\operatorname{cl}_{I^{\tau}}\left(p^{-1}\left(U_{i}\right)\right)$ is strongly $l$-embedded in $I^{\tau}$ and contains a strongly $l$-embedded copy of $I^{\tau}$. Hence, it follows from Corollary 3.5 and Corollary 3.7 that $C_{P}^{*}\left(\sum \mathrm{cl}_{I^{\tau}}\left(p^{-1}\left(U_{i}\right)\right)\right) \sim C_{P}^{*}\left(\sum I^{\tau}\right)$. On the other hand $U$ contains an $l^{*}$-embedded copy of $\sum I^{\tau}$ (see the proof of Theorem 2.15). Therefore, by Proposition 3.13, $C_{P}^{*}(U) \sim C_{P}^{*}\left(\sum I^{\tau}\right)$.

3.16 Theorem. Let $Y$ be one of the spaces $Q, I^{n}, \mu^{n}$ and $X$ be a locally compact subset of a $Y$-manifold. Then $C_{P}^{*}(X) \sim C_{P}^{*}\left(\sum Y\right)$ if $X$ contains a closed copy of $\sum Y$.

Proof. Let $X$ be a locally compact subspace of a $Y$-manifold $Z$ and let $X$ contain a closed copy of $\sum Y$. Then $C_{P}^{*}(X) \sim C_{P}^{*}\left(\sum Y\right) \times C_{P}^{*}\left(X ; \sum Y\right)$. Take an infinite locally finite open cover $\left\{V_{i} ; i \in N\right\}$ of $X$ such that each set $\mathrm{cl}_{X}\left(V_{i}\right)$ is compact and $\operatorname{cl}_{X}\left(V_{i}\right) \subset U_{i}$, where $U_{i}$ is an open subset of $Y$. Thus, each $\mathrm{cl}_{X}\left(V_{i}\right)$ is contained in a copy $Y_{i}$ of $Y$. Let $u: C_{P}\left(\sum \mathrm{cl}_{X}\left(V_{i}\right)\right) \rightarrow C_{P}(X)$ be a regular averaging operator for the natural mapping $p: \sum \mathrm{cl}_{X}\left(V_{i}\right) \rightarrow X$. As in the proof of Proposition 3.13, we get $\left(\prod C_{P}^{*}\left(\sum \mathrm{cl}_{X}\left(V_{i}\right)\right)\right)_{c}^{*} \sim\left(\prod C_{P}^{*}(X)\right)_{c}^{*} \times\left(\prod E\right)_{c}^{*}$, where $E$ is a linear subspace of $C_{P}^{*}\left(\sum \mathrm{cl}_{X}\left(V_{i}\right)\right)$. Since $\sum \mathrm{cl}_{X}\left(V_{i}\right)$ is a closed subset of $\sum Y_{i}$, by Corollary 3.3 we have $\left(\prod C_{P}^{*}\left(\sum Y_{i}\right)\right)_{c}^{*} \sim\left(\prod C_{P}^{*}\left(\sum \mathrm{cl}_{X}\left(V_{i}\right)\right)\right)_{c}^{*}$ $\times\left(\prod G\right)_{c}^{*}$, where $G=C_{P}^{*}\left(\sum Y_{i} ; \sum \mathrm{cl}_{X}\left(V_{i}\right)\right)$. Thus,

$$
\left(\prod C_{P}^{*}\left(\sum Y_{i}\right)\right)_{c}^{*} \sim\left(\prod C_{p}^{*}(X)\right)_{c}^{*} \times\left(\prod E\right)_{c}^{*} \times\left(\prod G\right)_{c}^{*} .
$$

Then

$$
\begin{aligned}
C_{P}^{*}(X) & \sim C_{P}^{*}\left(\sum Y\right) \times C_{P}^{*}\left(X ; \sum Y\right) \\
& \sim\left(\prod C_{P}^{*}\left(\sum Y\right)\right)_{c}^{*} \times C_{P}^{*}\left(X ; \sum Y\right)
\end{aligned}
$$


because $C_{P}^{*}\left(\sum Y\right) \sim\left(\prod C_{P}^{*}\left(\sum Y\right)\right)_{c}^{*}$ (see Corollary 3.5 and Proposition 3.11). Hence

$$
\begin{aligned}
C_{P}^{*}(X) & \sim\left(\prod C_{P}^{*}\left(\sum Y\right)\right)_{c}^{*} \times C_{P}^{*}\left(X ; \sum Y\right) \\
& \sim\left(\prod C_{P}^{*}\left(\sum Y\right)\right)_{c}^{*} \times C_{P}^{*}\left(\sum Y\right) \times C_{P}^{*}\left(X ; \sum Y\right) \\
& \sim\left(\prod C_{P}^{*}\left(\sum Y\right)\right)_{c}^{*} \times C_{P}^{*}(X) \\
& \sim C_{P}^{*}(X) \times\left(\prod C_{P}^{*}(X)\right)_{c}^{*} \times\left(\prod E\right)_{c}^{*} \times\left(\prod G\right)_{c}^{*} \\
& \sim\left(\prod C_{P}^{*}(X)\right)_{c}^{*} \times\left(\prod E\right)_{c}^{*} \times\left(\prod G\right)_{c}^{*} \\
& \sim\left(\prod C_{P}^{*}\left(\sum Y\right)\right)_{c}^{*} \sim C_{P}^{*}\left(\sum Y\right) .
\end{aligned}
$$

Added in proof. After this paper was submitted for publication Arhangel'skii [A4] introduced the notion of an $S$-stable space. A space $X$ is $S$-stable if $C_{P}(X) \sim C_{P}(X \times S)$, where $S=\{0,1 / n, n \in N\}$. Obviously, if $X \times S$ is a $k_{R}$-space, then $X$ is $S$-stable iff $\left(\prod C_{P}(X)\right)_{c} \sim C_{P}(X)$. An elementary proof of the $S$-stability of $\mu^{n}$ (without using Dranishnikov's results, see the proof of this fact in our Theorem 2.9) is given in [A4]. Arhangel'skii [A4] generalized our Theorem $2.8(\mathrm{ii})$ by proving that if a compact metric space $X$ contains a subspace $Y$ with $C_{P}(Y) \sim C_{P}(Q)$ then $C_{P}(X) \sim C_{P}(Q)$.

\section{REFERENCES}

[A1] A. V. Arhangel'skii, The principal of $\tau$-approximation and a test for equality of dimension of compact Hausdorff spaces, Soviet Math. Dokl. 21 (1980), 805-809.

[A2] $\quad-$ On linear homeomorphisms of function spaces, Soviet Math. Dokl. 25 (1982), 852855.

[A3] _,$O$ nekotoryh topologiceskih prostranstvah vstrečajuscihsja v funkcional' nom analize, Uspehi Mat. Nauk 31 (1978), 17-32.

[A4] _ On linear topological and topological classification of spaces $C_{p}(X)$, Zbornik Radova Filozofskog Fakulteta u Nišu, Ser. Matematika 3 (1989), 3-12.

[AČ] A. V. Arhangel'skii and M. M. Čoban, Funktional' nye vlojenija Tihonovskih prostranstv $i$ obobscennye retracty, preprint.

[BGM] J. Baars, J. de Groot and J. van Mill, Topological equivalence of certain function spaces II, VU (Amsterdam) report 321, December 1986.

[BGMP] J. Baars, J. de Groot, J. van Mill and J. Pelant, On topological and linear homeomorphisms of certain function spaces, University of Amsterdam, report 87-17, 1987.

[B] C. Bessaga, On topological classification of complete linear metric spaces, Fund. Math. 56 (1965), 251-288.

[Bt] M. Bestvina, Characterizing $k$-dimensional universal Menger compacta, Ph.D. thesis, The Univ. of Tennessee, Knoxville, 1984.

[Dr1] A. N. Dranishnikov, Absoljutnye F-znacnye retrakty $i$ prostranstva funkcii $v$ topologii potočečnoi shodimosti, Sibirsk Mat. J. 27 (1986), 74-86.

[Dr2] __, Universal' nye mengerovskie kompacty $i$ universal' nye otobrajenija, Mat. Sb. 129 (1986), 121-139. 
[D] J. Dugundji, An extension of Tietze's theorem, Pacific J. Math. 1 (1951), 353-367.

[GH] S. Gul' ko and T. Hmyleva, Kompaktnost' ne sohranjaetsja otnošeniem t-ekvivalentnosti, Mat. Zametki 39, 8 (1986), 895-903.

[KO] A. Koyama and T. Okada, On compacta which are l-equivalent to $I^{n}$, Tsukuba J. Math. 11, 1 (1987), 147-156.

[M] J. van Mill, Topological equivalence of certain function spaces, Compositio Math. 63 (1987), 159-188.

[N] N. Noble, The continuity of functions on Cartesian products, Trans. Amer. Math. Soc. 149 (1970), 187-198.

[Pv] D. Pavlovskii, On spaces of continuous functions, Soviet Math. Dokl. 22 (1980), 34-37.

[P] A. Pelczynski, Projections in certain Banach spaces, Studia Math. 19 (1960), 209-228.

[Ps] V. Pestov, The coincidence of the dimension dim of l-equivalent topological spaces, Soviet Math. Dokl. 28 (1982), 380-383.

[PP] E. Pol and R. Pol, Remarks on Cartesian products, Fund. Math. 93 (1976), 57-69.

Department of Mathematics, Sofia State University, 1126 Sofia, A. Ivanov 5, Bulgaria 